\title{
Gauss-Bonnet as effective cosmological constant
}

\author{
Liu Zhao, Kun Meng \\ School of Physics, Nankai university, Tianjin 300071, China \\ email:lzhao@nankai.edu.cn,kunmeng@mail.nankai.edu.cn
}

November 12, 2018

\begin{abstract}
It is known that Gauss-Bonnet terms in higher dimensional gravity can produce an effective cosmological constant. We add extra examples to this picture by presenting explicitly two branches of accelerating vacuum solutions to the Einstein-Gauss-Bonnet gravities with a bare cosmological constant in 5 and 6 dimensions. Both branches of solutions are of constant curvature and the effective cosmological constants are independent of the acceleration parameter. One branch (the "-" branch) of the solutions is well defined in the limit when the Gauss-Bonnet parameter approaches zero, in which case the effective cosmological constant becomes identical with the bare value, while the other (i.e. the "+") branch is singular in the same limit, and beyond this singular limit, the effective cosmological constant is inversely proportional to the Gauss-Bonnet parameter with a negative constant of proportionality when the bare value vanishes.
\end{abstract}

\section{Introduction}

Gravitational theories with higher curvature terms have attracted considerable attention. Among these, the Lovelock family [1] of gravity theories - of which EinsteinGauss-Bonnet (EGB) gravity is a member involving contributions in curvature tensor up to second order - are of particular interests because these are the only theories which degenerate naturally into Einstein gravity in 4-dimensions [2]. Exact static spherically symmetric black hole solutions of EGB gravity have been found in Refs. [3, 4], and of the Einstein-Maxwell-Gauss-Bonnet (EMGB) and Born-Infeld-Gauss-Bonnet models in Refs. [5, 6]. Black hole solutions with nontrivial topology have also been studied in $[7,8,9,10,11]$. Other solutions like charged rotating black brane solutions [12], TaubNUT solutions in EMGB gravity in $2 k+2$ dimensions [13], nonstatic brane cosmology solution [14] and numeric black hole solutions [15] have also been studied. The thermodynamics of various black holes have also been studied in Refs. [5, 6, 16, 17, 18, 19, 20].

In this paper, we shall consider another aspect of EGB gravity, i.e. the effect of Gauss-Bonnet term on producing effective cosmological constant. That the Gauss- 
Bonnet term can produce effective cosmological constant has been known for a long time, see, e.g. [3]. In [8] and [11], the same effect were also reproduced. Unlike the solutions given in [3] [8] [11], the explicit solutions we shall present is written in a special form which is conformal to the standard (A)dS metrics and the conformal factor depends on an angular coordinate, thus making the spacetime anisotropic. Similar anisotropic vacuum solutions in Einstein gravity were studied in [21] and are known to represent accelerating vacua. We believe that such vacuum solutions for EGB gravities were not discussed before in the literatures.

\section{Einstein vacua for Gauss-Bonnet gravity in 5- dimensions}

We begin by writing down the action of EGB gravity in $n$-dimensions:

$$
\begin{aligned}
& I=\frac{1}{16 \pi G} \int \mathrm{d}^{n} x \sqrt{-g}\left[R-2 \Lambda+\xi \mathcal{L}_{\mathrm{GB}}\right], \\
& \mathcal{L}_{\mathrm{GB}}=R_{\mu \nu \gamma \delta} R^{\mu \nu \gamma \delta}-4 R_{\mu \nu} R^{\mu \nu}+R^{2},
\end{aligned}
$$

where $G$ is the $n$-dimensional Newton constant, $\Lambda$ is the bare cosmological constant and $\xi$ is the Gauss-Bonnet parameter.

The field equation that follow from the action reads

$$
R_{\mu \nu}-\frac{1}{2} g_{\mu \nu} R+\Lambda g_{\mu \nu}+\xi H_{\mu \nu}=0
$$

where

$$
H_{\mu \nu}=2\left(R_{\mu \lambda \rho \sigma} R_{\nu}{ }^{\lambda \rho \sigma}-2 R_{\mu \rho \nu \sigma} R^{\rho \sigma}-2 R_{\mu \sigma} R_{\nu}{ }^{\sigma}+R R_{\mu \nu}\right)-\frac{1}{2} \mathcal{L}_{\mathrm{GB}} g_{\mu \nu}
$$

In this section, we shall consider the case $n=5$. Using the metric ansatz

$$
\mathrm{d} s^{2}=\frac{1}{\alpha^{2}(x+y)^{2}}\left[-Y(y) \mathrm{d} t^{2}+\frac{\mathrm{d} y^{2}}{Y(y)}+\frac{\mathrm{d} x^{2}}{X(x)}+X(x)\left(\frac{\mathrm{d} z^{2}}{Z(z)}+Z(z) \mathrm{d} \phi^{2}\right)\right]
$$

and by use of some computer algebra, we found that the following are exact solutions to the equations of motion (1)-(2):

$$
\begin{aligned}
& X(x)=\frac{1}{2} C_{1} x^{2}+C_{2} x+C_{3}, \\
& Y(y)=-\left(\frac{1}{2} C_{1} y^{2}-C_{2} y+C_{3}\right)+\frac{1}{12 \alpha^{2} \xi}(3+\delta \sqrt{12 \xi \Lambda+9}), \\
& Z(z)=\frac{1}{2}\left(C_{1} C_{3}-\frac{1}{2} C_{2}^{2}\right) z^{2}+C_{4} z+C_{5},
\end{aligned}
$$

where $\delta= \pm 1, C_{1} \sim C_{5}$ are integration constants and $\alpha$ is a free parameter introduced in the metric ansatz. We can freely take $C_{2}=C_{4}=0$ and $\operatorname{abs}\left(C_{3}\right)=1$ by use of the 
coordinate shift degrees of freedom. Further more, by use of the coordinate rescaling degrees of freedom, we can make $\operatorname{abs}\left(C_{1}\right)=2$. We also choose $C_{1} C_{3}=-2$ and $C_{5}=1$ for simplicity. Then the solutions will read

$$
\begin{aligned}
& X(x)=\epsilon\left(1-x^{2}\right) \\
& Y(y)=\epsilon\left(y^{2}-1\right)+\frac{1}{12 \alpha^{2} \xi}(3+\delta \sqrt{12 \xi \Lambda+9}) \\
& Z(z)=1-z^{2}
\end{aligned}
$$

where $\epsilon= \pm 1$ and is independent of $\delta$.

The above vacuum solutions were written in an unfamiliar coordinate system. To make them more recognizable, we need to make some coordinate transformations. For all values of $\epsilon$, we change $y$ and $z$ as follows,

$$
\begin{aligned}
& t \rightarrow \tau=\frac{t}{\alpha}, \\
& y \rightarrow r=\frac{1}{\alpha y}, \\
& z \rightarrow \theta_{2}=\arccos (z) .
\end{aligned}
$$

The $x$ coordinate transformation will depend on the value of $\epsilon$. For $\epsilon=1$, we take the transformation

$$
x \rightarrow \theta_{1}=\arccos (-x),
$$

and for the case $\epsilon=-1$, we take

$$
x \rightarrow \theta_{1}=\operatorname{arccosh}(-x) .
$$

In all cases the coordinates $t$ and $\phi$ are kept unchanged. The resulting metrics after the above transformations are given as follows. For $\epsilon=+1$, we have

$$
\begin{aligned}
d s^{2} & =\frac{1}{\left(1-\alpha r \cos \theta_{1}\right)^{2}}\left(-f_{1}(r) d \tau^{2}+\frac{1}{f_{1}(r)} d r^{2}+r^{2} d \Omega_{3+}^{2}\right), \\
f_{1}(r) & =1-\frac{\kappa_{1} r^{2}}{\ell_{1}^{2}} \equiv 1-r^{2}\left(\alpha^{2}-\frac{3+\delta \sqrt{12 \xi \Lambda+9}}{12 \xi}\right), \\
d \Omega_{3+}^{2} & =d \theta_{1}^{2}+\sin ^{2} \theta_{1}\left(d \theta_{2}^{2}+\sin ^{2} \theta_{2} d \phi^{2}\right),
\end{aligned}
$$

where $\kappa_{1}= \pm 1$ depending on the values of $\delta, \xi, \Lambda$ and $\alpha$. Similarly, for $\epsilon=-1$, the corresponding metric becomes

$$
\begin{aligned}
d s^{2} & =\frac{1}{\left(\alpha r \cosh \theta_{1}-1\right)^{2}}\left(-f_{2}(r) d \tau^{2}+\frac{1}{f_{2}(r)} d r^{2}+r^{2} d \Omega_{3-}^{2}\right), \\
f_{2}(r) & =-1+\frac{\kappa_{2} r^{2}}{\ell_{2}^{2}} \equiv-1+r^{2}\left(\alpha^{2}+\frac{3+\delta \sqrt{12 \xi \Lambda+9}}{12 \xi}\right), \\
d \Omega_{3-}^{2} & =d \theta_{1}^{2}+\sinh ^{2} \theta_{1}\left(d \theta_{2}^{2}+\sin ^{2} \theta_{2} d \phi^{2}\right),
\end{aligned}
$$


where, again, $\kappa_{2}= \pm 1$ depending on the values of $\delta, \xi, \Lambda$ and $\alpha$.

The metric (7) is explicitly conformal to the standard (A)dS metric. Without much effort, it can be shown that, although we obtained the metric (7) as an exact solution to the equations of motion (1) (with the insertion of (2)) of EGB gravity, it is indeed also a solution to the pure Einstein equation

$$
R_{\mu \nu}-\frac{1}{2} g_{\mu \nu} R+\Lambda_{\mathrm{eff}} g_{\mu \nu}=0
$$

with the effective cosmological constant $\Lambda_{\text {eff }}$ given as

$$
\Lambda_{\text {eff }}=6\left(\frac{\kappa_{1}}{\ell_{1}^{2}}-\alpha^{2}\right)=-\frac{3+\delta \sqrt{12 \xi \Lambda+9}}{2 \xi} .
$$

The form of the metric (7) has already been found in the previous work [21] by one of the authors as an exact solution of Einstein gravity with non vanishing cosmological constant.

Apart from the overall conformal factor, the metric (10) is identical to the well known topological AdS black hole metric [22, 23, 24] with mass set to zero and curvature of spacial sections set to negative constants. The complete metric (10) also satisfies the effective Einstein equation (13) with the effective cosmological constant given by

$$
\Lambda_{\mathrm{eff}}=6\left(\alpha^{2}-\frac{\kappa_{2}}{\ell_{2}^{2}}\right)=-\frac{3+\delta \sqrt{12 \xi \Lambda+9}}{2 \xi} .
$$

Thus both the cases $\epsilon=+1$ and $\epsilon=-1$ correspond to the same effective cosmological constant.

For both the metric (7) or the metric (10), it can be easily checked that the parameter $\alpha$ has a very clear physical meaning. It corresponds to the magnitude of proper acceleration of the origin of the coordinate system being used. It should be remarked that accelerating vacua with conformal factor as given in (10) (i.e. involving hyperbolic cosine in the denominator) have not been found before.

It follows from (14)-(15) that the effective cosmological constant is independent of the acceleration parameter. Actually, by taking the $\alpha=0$ limit in (7), the effective cosmological constant can be readily read off because the metric falls back to that of the standard (A)dS. This is in sharp contrast to the case of Einstein gravity, for which it is known that the presence of the accelerating parameter $\alpha$ will shift the corresponding cosmological constant [21].

To see how the dependence on the bare cosmological constant $\Lambda$, the Gauss-Bonnet parameter $\xi$ and the signature $\delta$ affects the value of the effective cosmological constant, it is necessary to take various limits. First we consider the "-" branch (i.e. $\delta=-1$ ). In this case $\Lambda_{\text {eff }}$ is well behaved in the limit $\xi \rightarrow 0$, yielding

$$
\lim _{\xi=0, \delta=-1} \Lambda_{\text {eff }}=\Lambda
$$


On the other hand, the "+" branch does not have a well defined limit while $\xi \rightarrow 0$. So, we may consider another limit, i.e. $\Lambda \rightarrow 0$. The limiting value of $\Lambda_{\text {eff }}$ in this case reads

$$
\lim _{\Lambda=0} \Lambda_{\text {eff }}=-\frac{3}{2 \xi}(1+\delta)
$$

which is nonvanishing only for $\delta=+1$. Therefore, in order to produce a nonzero effective constant starting from a vanishing bare value, we need to take $\delta=+1$, and the corresponding effective cosmological constant is

$$
\Lambda_{\mathrm{eff}}=-\frac{3}{\xi}
$$

\section{Effective cosmological constant in $6 \mathrm{D}$}

In the last section we have seen that the presence of the Gauss-Bonnet parameter $\xi$ can produce an effective cosmological constant in 5-dimensions. It is natural to ask whether this is an accidental fact in $5 \mathrm{D}$ or is a general property in all higher dimensions. In this section, we shall try to partially settle this question by presenting explicit results in the case of 6 -dimensions.

We start by mimicking the ansatz (3) by writing down the following metric:

$$
\begin{aligned}
\mathrm{d} s^{2}= & \frac{1}{\alpha^{2}(x+y)^{2}} \\
& \times\left\{-Y(y) \mathrm{d} t^{2}+\frac{\mathrm{d} y^{2}}{Y(y)}+\frac{\mathrm{d} x^{2}}{X(x)}+X(x)\left[\frac{\mathrm{d} z^{2}}{Z(z)}+Z(z)\left(\frac{\mathrm{d} w^{2}}{W(w)}+W(w) \mathrm{d} \phi^{2}\right)\right]\right\} .
\end{aligned}
$$

Inserting into the equation of motion (1)-(2), we get the following solution,

$$
\begin{aligned}
X(x) & =\frac{1}{2} C_{1} x^{2}+C_{2} x+C_{3}, \\
Y(y) & =-\left(\frac{1}{2} C_{1} y^{2}-C_{2} y+C_{3}\right)+\frac{1}{60 \alpha^{2} \xi}(5+\delta \sqrt{60 \xi \Lambda+25}), \\
Z(z) & =\frac{1}{2}\left(C_{1} C_{3}-\frac{1}{2} C_{2}^{2}\right) z^{2}+C_{4} z+C_{5}, \\
W(w) & =\frac{1}{2}\left(C_{1} C_{3} C_{5}-\frac{1}{2} C_{4}^{2}-\frac{1}{2} C_{2}^{2} C_{5}\right) w^{2}+C_{6} w+C_{7},
\end{aligned}
$$

where $\delta= \pm 1$ and $C_{1} \sim C_{7}$ are integration constants. By properly choosing the values of these constants using various coordinate choice freedoms, we can simplify the solution into the form

$$
\begin{aligned}
X(x) & =\epsilon\left(1-x^{2}\right) \\
Y(y) & =\epsilon\left(y^{2}-1\right)+\frac{1}{60 \alpha^{2} \xi}(5+\delta \sqrt{60 \xi \Lambda+25}), \\
Z(z) & =1-z^{2} \\
W(w) & =1-w^{2} .
\end{aligned}
$$


One can proceed as in the previous section to rewrite the solution in a form which is conformal to the (A)dS metric in the standard form. However, omitting the details, we jump to the conclusion that the above metric is also a solution to the Einstein equation (13) with the effective cosmological constant given by

$$
\Lambda_{\mathrm{eff}}=-\frac{5+\delta \sqrt{60 \xi \Lambda+25}}{6 \xi} .
$$

For vanishing $\Lambda, \Lambda_{\text {eff }}$ is nonzero only in the "+" branch and the corresponding value is

$$
\Lambda_{\mathrm{eff}}=-\frac{5}{3 \xi}
$$

\section{Discussion}

We have shown that EGB gravities in 5 and 6 dimensions possess accelerating vacua which are identical to Einstein vacua with effective cosmological constants. The solutions can be divided into two branches by choosing different signatures $\delta$, just like in the case in the absence of the acceleration parameter [3]. The "-" branch is well behaved when the Gauss-Bonnet parameter $\xi \rightarrow 0$, in which case the effective cosmological constant approaches the bare value. In contrast, the "+" branch is not well behaved in the limit $\xi \rightarrow 0$, and for $\xi \neq 0$ the effective cosmological constant does not vanish even the bare value is zero. In both $5 \mathrm{D}$ and $6 \mathrm{D}$ cases the effective cosmological constant in the "+" branch with vanishing bare value is inversely proportional to the Gauss-Bonnet parameter, with the constant of proportionality being a negative number. Thus, for instance, if we need a small and positive effective cosmological constant, the Gauss-Bonnet parameter must be negative and have a very large absolute value. On the other hand, if the Gauss-Bonnet parameter were taken to be positive, the effective cosmological constant will be negative.

It is remarkable that the effective cosmological constants does not depends on the acceleration parameter (as apposed to the case of Einstein gravity), and the concrete values we get in $5 \mathrm{D}$ and $6 \mathrm{D}$ agree with the corresponding values in the non accelerating cases [3]. We speculate that these properties may continue to hold in even higher dimensions. It is interesting to ask whether higher order Lovelock parameters can also produce effective cosmological constants. We leave this question for later considerations.

In closing this article, let us pay some words toward the similar effects in pure GB gravity in the absence of Einstein term. It is not quite difficult to check that the solution (4)-(6), with (5) replaced by

$$
Y(y)=\epsilon\left(y^{2}-1\right)+\frac{1}{6 \alpha^{2} \xi}(\delta \sqrt{3 \xi \Lambda})
$$

satisfies the equation of motion for the so-called pure GB gravity in 5D,

$$
\Lambda g_{\mu \nu}+\xi H_{\mu \nu}=0 \text {. }
$$


Similarly, the solution (16)-(19), with $Y(y)$ replaced by

$$
Y(y)=\epsilon\left(y^{2}-1\right)+\frac{1}{30 \alpha^{2} \xi}(\delta \sqrt{15 \xi \Lambda}),
$$

also satisfy the equation of motion for pure GB gravity in 6D. The corresponding solutions are again Einstein vacua, with the effective cosmological constants given by

$$
\Lambda_{\mathrm{eff}}=-\frac{\delta \sqrt{3 \xi \Lambda}}{\xi}
$$

in the $5 \mathrm{D}$ case and

$$
\Lambda_{\mathrm{eff}}=-\frac{\delta \sqrt{15 \xi \Lambda}}{3 \xi}
$$

in the 6D case. So it is clear that for pure GB gravity, the effective cosmological constants will approach zero in both branches of accelerating vacua if the bare value vanishes.

\section{Acknowledgment}

This work is supported by the National Natural Science Foundation of China (NSFC) through grant No.10875059. L.Z. would like to thank the organizer and participants of "The advanced workshop on Dark Energy and Fundamental Theory" supported by the Special Fund for Theoretical Physics from the National Natural Science Foundation of China with grant no: 10947203 for discussions.

\section{References}

[1] D. Lovelock, "The Einstein tensor and its generalizations," J. Math. Phys.12 (1971) 498.

[2] D. Lovelock, "The Four-Dimensionality of Space and the Einstein Tensor," J. Math. Phys.13 (1972) 874.

[3] D. Boulware and S. Deser, "String-generated gravity models," Phys. Rev. Lett. (1985).

[4] J. T. Wheeler, "Symmetric solutions to the Gauss-Bonnet extended Einstein equations," Nucl. Phys. B268 (1986) 737.

[5] D. L. Wiltshire, "Spherically symmetric solutions of Einstein-Maxwell theory with a Gauss-Bonnet term", Phy. Lett. B V169, No.1 (1986) 36-40.

[6] I. P. Neupane, "Black hole entropy in string-generated gravity models," Phys.Rev. D67: 061501, 2003 [arXiv: hep-th/0212092]. 
[7] R.-G. Cai and K.-S. Soh, "Topological black holes in the dimensionally continued gravity," Phys. Rev. D59: 044013, 1999 [arXiv: gr-qc/9808067].

[8] R.-G. Cai, "Gauss-Bonnet Black Holes in AdS Spaces," Phys.Rev.D65: 084014, 2002 [arXiv: hep-th/0109133].

[9] R. Aros, R. Troncoso, and J. Zanelli, "Black holes with topologically nontrivial AdS asymptotics," Phys. Rev. D63: 084015, 2002 [arXiv: hep-th/0011097].

[10] Y. M. Cho and I. P. Neupane, "Anti-de Sitter Black Holes, Thermal Phase Transition and Holography in Higher Curvature Gravity," Phys. Rev. D66: 024044, 2002 [arXiv: hep-th/0202140].

[11] M. H. Dehghani, "Asymptotically (anti)-de Sitter solutions in Gauss-Bonnet gravity without a cosmological constant," Phys. Rev. D70: 064019, 2004 [arXiv: hep-th/0405206].

[12] M. H. Dehghani, "Charged Rotating Black Branes in anti-de Sitter Einstein-Gauss-Bonnet Gravity," Phys. Rev. D67: 064017, 2003 [arXiv: hep-th/0211191].

[13] M. H. Dehghani and S. H. Hendi, "Taub-NUT/Bolt Black Holes in Gauss-Bonnet-Maxwell Gravity," Phys. Rev. D73: 084021, 2006 [arXiv: hep-th/0602069].

[14] C. Charmousis and J.-F. Dufaux, "General Gauss-Bonnet brane cosmology," Class. Quant. Grav. 19 (2002) 4671-4682 [arXiv: hep-th/0202107].

[15] Y. Brihaye, "Charged, rotating black holes in Einstein-Gauss-Bonnet gravity," arXiv: 1108.2779.

[16] R. Myers and J. Simon, "Black-hole thermodynamics in Lovelock gravity," Phys. Rev. D38, No.8 (1988) 2434.

[17] R. Myers, "Superstring gravity and black holes," Nucl. Phys. B289 (1987) 701.

[18] M. Cvetic, S. Nojiri, and S. D. Odintsov, "Black Hole Thermodynamics and Negative Entropy in deSitter and Anti-de Sitter Einstein-Gauss-Bonnet gravity," Nucl. Phys. B628: 295-330, 2002 [arXiv: hep-th/0112045].

[19] S. Nojiri and S. D. Odintsov, "Anti-de Sitter Black Hole Thermodynamics in Higher Derivative Gravity and New Confining-Deconfining Phases in dual CFT," Phys. Lett. B521: 87-95, 2001; Erratum-ibid. B542: 301, 2002 [arXiv: hep-th/0109122].

[20] Y. Cho and I. Neupane, "Anti-de Sitter black holes, thermal phase transition, and holography in higher curvature gravity," Phys. Rev. D66: 024044, 2002.

[21] L. Zhao, "Note on a class of anisotropic Einstein metrics," arXiv: 1106.5027]. 
[22] D. R. Brill and J. Louko, "Thermodynamics of (3+1)-dimensional black holes with toroidal or higher genus horizons", Phys. Rev. D56 (1997) 3600-3610 [arXiv: gr-qc/9705012].

[23] R. Emparan, "AdS Membranes Wrapped on Surfaces of Arbitrary Genus," Phys. Lett. B432 (1998) 74-82 [arXiv: hep-th/9804031].

[24] D. Birmingham, "Topological Black Holes in Anti-de Sitter Space", Class. Quant. Grav. 16 (1999) 1197-1205 [arXiv: hep-th/9808032]. 